\section{Evaluation of the association between anticarbamylated protein antibodies and the longitudinal course of functional ability in rheumatoid arthritis}

Clinically relevant joint destruction has now become infrequent in patients with newly diagnosed rheumatoid arthritis (RA), while other disease outcomes such as functional ability and disease persistence have become more important. Recently, novel auto-antibody reactivities have been identified in RA, with the ultimate aim to better characterise patients with RA. Anticarbamylated protein (anti-CarP) antibodies were recently identified and associated with more severe radiographic progression, ${ }^{1}$ but the association with other outcomes was unexplored. With great interest we read the study by Humphreys et al, ${ }^{2}$ reporting more severe functional disability in anti-CarP positive patients with inflammatory polyarthritis. According to the reported unadjusted $\beta$-coefficient $(95 \% \mathrm{CI})$, patients with anti-CarP antibodies had $0.21(0.14$ to 0.29$)$ higher scores on the Health Assessment Questionnaire (HAQ) during a median follow-up duration of 8 years. This association was statistically independent of the presence of anticitrullinated peptide antibodies (ACPA) and rheumatoid factor (RF). In general replication serves to validate occasional findings. Therefore we studied the association between anti-CarP antibodies and the course of functional disability in 1604 patients with RA who were included in two observational cohorts and had 6474 HAQ measurements over time.

A total of 784 patients with RA were included in the Swedish Better Anti-Rheumatic PharmacOTherapy (BARFOT) cohort $^{3}$ between 1993 and 1999, and 820 patients in the Leiden Early Arthritis Clinic (EAC) ${ }^{4}$ between 1993 and 2010 (table 1). Both cohorts are described elsewhere. ${ }^{3}{ }^{4}$ Linear mixed models with HAQ as continuous outcome and anti-CarP status assessed in baseline serum (as described previously) ${ }^{1}$ as predictor were performed. Adjustments were done for age, gender and initial treatment strategies as described previously. ${ }^{5}$ To examine if the association was independent of the effects of other auto-antibodies, additional adjustments were made for ACPA and RF status.

Of the patients with RA, $36 \%$ and $46 \%$ were positive for anti-CarP antibodies in the BARFOT and Leiden EAC cohorts, respectively (table 1). Univariate analysis within the BARFOT

\begin{tabular}{lll}
\hline Table $1 \quad$ Patient characteristics & & \\
\hline & BARFOT & Leiden EAC \\
Total patients (n) & 784 & 820 \\
Total HAQ assessments (n) & 3951 & 2523 \\
Maximum follow-up (years) & 8 & 5 \\
Year of diagnosis & $1993-1999$ & $1993-2010$ \\
Female, $\mathrm{n}(\%)$ & $515(65.7)$ & $550(67.1)$ \\
Age at inclusion in years, mean (SD) & $57.6(15.6)$ & $56.6(15.6)$ \\
Auto-antibodies & & \\
$\quad$ anti-CarP positive, $\mathrm{n}(\%)$ & $278(35.5)$ & $374(45.6)$ \\
$\quad$ ACPA positive, $\mathrm{n}(\%)$ & $452(57.7)$ & $423(51.6)$ \\
RF positive, $\mathrm{n}(\%)$ & $443(56.5)$ & $474(57.8)$
\end{tabular}

ACPA, anticitrullinated peptide antibodies; anti-CarP, anticarbamylated protein antibodies; BARFOT, Better Anti-Rheumatic PharmacOTherapy; EAC, Early Arthritis Clinic; $\mathrm{HAQ}$, health assessment questionnaire; RF, rheumatoid factor.

Within the BARFOT cohort, HAQ was assessed at 6 months, 1, 2, 5 and 8 years. Within the EAC cohort, HAQ was assessed yearly up to 5 years of follow-up. cohort showed that anti-CarP positivity was significantly associated with functional disability; patients carrying anti-CarP antibodies had 0.082 (95\% CI 0.0064 to 0.16 , p value 0.034 ) higher HAQ scores during the 8 years of follow-up compared with patients without anti-CarP antibodies (figure 1A). In the model including all three auto-antibodies, anti-CarP was not significantly associated with HAQ over time: effect size of anti-CarP $0.053,95 \%$ CI -0.039 to $0.15, \mathrm{p}$ value 0.26 . Also ACPA and RF did not associate with HAQ over time independent of the other auto-antibodies ( $p$ values, respectively, 0.29 and 0.94). Within the Leiden EAC, anti-CarP was not associated with functional ability during 5 years of disease: effect size $0.026,95 \%$ CI -0.053 to $0.10, \mathrm{p}$ value 0.52 ; after additional adjustment for ACPA and RF, the effect size of the association was $0.0051,95 \% \mathrm{CI}-0.094$ to $0.10, \mathrm{p}$ value 0.92 (figure $1 \mathrm{~B}$ ).

Thus, in contrast to the finding of Humphreys et al, we did not observe that anti-CarP was associated with functional ability, independent of ACPA and RF. Unadjusted analysis in the BARFOT showed an association. However, the effect size of 0.082 was below the minimal clinical important difference (MCID), which is reported to range between 0.09 and 0.38 for the HAQ. ${ }^{6}$ Although the effect size observed in the Norfolk Arthritis Register (NOAR) cohort was in this range of MCID, the effects observed in our cohorts were below this limit. In conclusion, we could not replicate that the presence of anti-CarP antibodies is associated with a clinically meaningful difference in functional ability in RA.
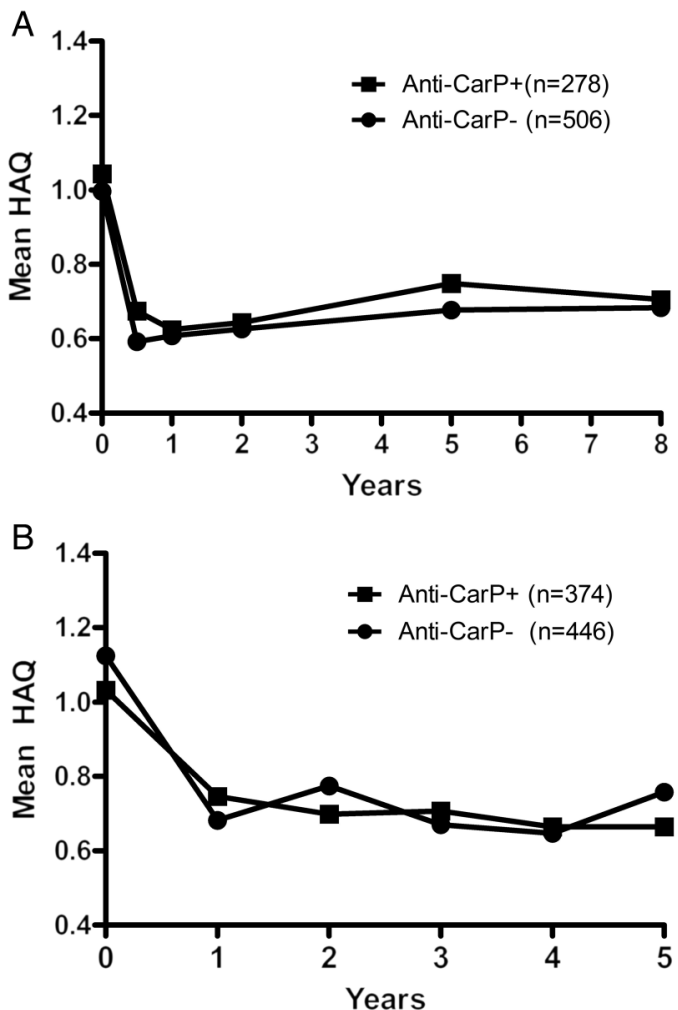

Figure 1 Health Assessment Questionnaire (HAQ) scores during the disease course according to presence of anticarbamylated protein (anti-CarP) antibodies in patients with rheumatoid arthritis (RA) from the (A) Better Anti-Rheumatic PharmacOTherapy (BARFOT) and (B) Leiden Early Arthritis Clinic (EAC) cohorts. Presented are observed mean HAQ scores assessed in (A) 784 patients with RA over 8 years from the BARFOT cohort and in (B) 820 patients with RA over 5 years from the Leiden EAC cohort. 


\section{S Ajeganova, ${ }^{1,2}$ B Svensson, ${ }^{3}$ T W J Huizinga, ${ }^{2}$ A H M van der Helm-van Mil, $^{2} \mathrm{H}$ W van Steenbergen ${ }^{2}$}

${ }^{1}$ Rheumatology Unit, Department of Medicine, Karolinska Institutet at Karolinska University Hospital Huddinge, Stockholm, Sweden

${ }^{2}$ Department of Rheumatology, Leiden University Medical Center, Leiden, The Netherlands

${ }^{3}$ Department of Clinical Sciences, Section of Rheumatology, Lund University, Lund, Sweden

Correspondence to Dr Sofia Ajeganova, Rheumatology Unit R92, Department of Medicine, Karolinska Institutet at Karolinska University Hospital Huddinge, Stockholm 14186, Sweden; sofia.ajeganova@ki.se

Contributors All authors were involved in drafting the manuscript or in revising it critically for important intellectual content. All authors approved the final version to be published.

Funding This work was supported by a Vidi grant of the Netherlands Organisation for Scientific Research, the Dutch Arthritis Foundation, the IMI JU funded project BeTheCure and the Swedish Rheumatism Association.

Competing interests TWJH is on a patent application for the use of anticarbamylated protein antibodies in diagnostics.

Patient consent Obtained.

Ethics approval Local ethics committees.

Provenance and peer review Not commissioned; internally peer reviewed.

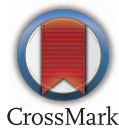

To cite Ajeganova S, Svensson B, Huizinga TWJ, et al. Ann Rheum Dis 2016;75: e14.

Received 28 November 2015

Accepted 29 November 2015

Published Online First 23 December 2015

\section{Linked}

http://dx.doi.org/10.1136/annrheumdis-2015-208957

Ann Rheum Dis 2016;75:e14. doi:10.1136/annrheumdis-2015-208954

\section{REFERENCES}

1 Shi J, Knevel R, Suwannalai $P$, et al. Autoantibodies recognizing carbamylated proteins are present in sera of patients with rheumatoid arthritis and predict joint damage. Proc Natl Acad Sci 2011;108:17372-7.

2 Humphreys JH, Verheul MK, Barton A, et al. Anticarbamylated protein antibodies are associated with long-term disability and increased disease activity in patients with early inflammatory arthritis: results from the Norfolk Arthritis Register. Ann Rheum Dis 2015. Published Online First: 6 Oct 2015. doi:10.1136/ annrheumdis-2015-207326

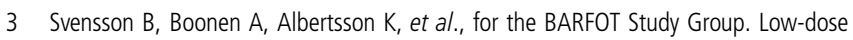
prednisolone in addition to the initial disease-modifying antirheumatic drug in patients with early active rheumatoid arthritis reduces joint destruction and increases the remission rate: A two-year randomized trial. Arthritis Rheum 2005;52:3360-70.

4 De Rooy DP, van der Linden MP, Knevel R, et al. Predicting arthritis outcomes-what can be learned from the Leiden Early Arthritis Clinic? Rheumatology 2011; 50:93-100.

5 Ajeganova S, Steenbergen van HW, Nies JAB van, et al. Disease-modifying antirheumatic drug-free sustained remission in rheumatoid arthritis: an increasingly achievable outcome with subsidence of disease symptoms. Ann Rheum Dis 2015. Published Online First: 13 May 2015. doi:10.1136/annrheumdis-2014-207080

6 Pope JE, Khanna D, Norrie $D$, et al. The minimally important difference for the health assessment questionnaire in rheumatoid arthritis clinical practice is smaller than in randomized controlled trials. J Rheumatol 2009;36:254-9.

7 Ward MM, Guthrie LC, Alba MI. Clinically important changes in individual and composite measures of rheumatoid arthritis activity: thresholds applicable in clinical trials. Ann Rheum Dis 2015;74:1691-6. 